This is the submitted version of the article:

Fariña D., Álvarez M., Márquez S., Dominguez C., Lechuga L.M.. Sensitivity analysis for improving nanomechanical photonic transducers biosensors. J ournal of Physics D: Applied Physics, (2015). 48. 335401: - . 10.1088/0022-3727/48/33/335401.

Available at:

https://dx.doi.org/10.1088/0022-3727/48/33/335401 


\title{
Sensitivity analysis for improving nanomechanical photonic transducers biosensors
}

\author{
D. Fariña ${ }^{1,2}$, Mar Álvarez ${ }^{2,1}$, S. Márquez ${ }^{2,1}$, Carlos Dominguez $^{3}$ and Laura M. \\ Lechuga $^{2,1^{*}}$ \\ ${ }^{1}$ CIBER-BBN Networking Center on Bioengineering, Biomaterials and Nanomedicine., Spain \\ ${ }^{2}$ Nanobiosensors and Bioanalytical Applications Group, Institut Catala de Nanociencia i Nanotecnologia (ICN2), \\ CSIC, 08193, Bellaterra, Barcelona, Spain \\ ${ }^{3}$ Microelectronics Institute of Barcelona (IMB-CNM), CSIC, Barcelona, Spain \\ laura.lechuga@cin2.es
}

\begin{abstract}
The achievement of high sensitivity and highly integrated transducers is one of the main challenges in the development of highthroughput biosensors. The aim of this study is to improve the final sensitivity of an opto-mechanical device to be applied as a reliable biosensor. We report an analysis of the mechanical and optical properties of optical waveguide microcantilevers transducers, and its dependency with the device design and dimensions. The selected layout (geometry) based in two butt-coupled misaligned waveguides display better sensitivities than an aligned one. With this configuration, we find that an optimal microcantilever thickness range between $150 \mathrm{~nm}$ and $400 \mathrm{~nm}$, would increase both the microcantilever bending during the biorecognition process and the optical sensitivity. Moreover, the analysis show that single mode behaviour of the propagating radiation is required to avoid the modal interference that could misunderstand the readout signal.
\end{abstract}

(C)2015 Optical Society of America

OCIS codes: (200.4880) Optomechanics; (000.4430) Numerical approximation and analysis; (130.3120) Integrated optics devices; (280.1415) Biological sensing and sensors; (280.4788) Optical sensing and sensors.

\section{References and links}

1. M. Alvarez and L. M. Lechuga, "Microcantilever-based platforms as biosensing tools", Analyst 135, 827-836 (2010).

2. J. W. Noh, R. R. Anderson, S. Kim, W. Hu, and G. P. Nordin, "In-plane all-photonic transduction with differential splitter using double-step rib waveguide for photonic microcantilever arrays", Opt. Express 17, 20012-20020 (2009).

3. J. W. Noh, R. R. Anderson, S. Kim, W. Hu, and G. P. Nordin, "Sensitivity enhancement of differential splitterbased transduction for photonic microcantilever arrays", Nanotechnology 21, 155501 (2010).

4. R. Panergo, C.-S. Huang, C.-S. Liu, P. G. Reinhall, and W.-C. Wang, "Resonant Polymeric Waveguide Cantilever Integrated for Optical Scanning", J. Light. Technol. 25, 850-860 (2007).

5. A. Llobera, V. J. Cadarso, K. Zinoviev, C. Dominguez, S. Buttgenbach, J. Vila, J. A. Plaza, and S. Biittgenbach, "Poly(Dimethylsiloxane) Waveguide Cantilevers for Optomechanical Sensing", Photonics Technol. Lett. IEEE 21, 79-81 (2009).

6. S. T. Koev, R. Fernandes, W. E. Bentley, and R. Ghodssi, "A cantilever sensor with an integrated optical readout for detection of enzymatically produced homocysteine", IEEE Trans. Biomed. Circuits Syst. 3, 415-423 (2009).

7. M. W. Pruessner, T. H. Stievater, M. S. Ferraro, W. S. Rabinovich, J. L. Stepnowski, and R. A. McGill, "Waveguide micro-opto-electro-mechanical resonant chemical sensors", Lab. Chip 10, 762-768 (2010).

8. J. E. Sader, "Surface stress induced deflections of cantilever plates with applications to the atomic force microscope: Rectangular plates", J. Appl. Phys. 89, 2911 (2001).

9. A. N. Cleland, Foundations of Nanomechanics, 1st ed. (Springer, 2002).

10. M. Alvarez, J. Tamayo, J. A. Plaza, K. Zinoviev, C. Domínguez, and L. M. Lechuga, "Dimension dependence of the thermomechanical noise of microcantilevers", J. Appl. Phys. 99, 1-7 (2006).

11. S. Timoshenko, Vibration Problems in Engineering, 2nd ed. (London; printed in U.S.A., 1937).

12. Hosaka H., Itao K., and Kuroda S., "Damping characteristics of beam-shaped micro-oscillators", Sens. Actuators Phys. 49, 87-95 (1995). 
13. R. G. Hunsperger, Integrated Optics: Theory and Technology (Springer, 2009).

14. "Comsol Multiphysics," http://www.comsol.com/.

15. "SciPy," http://www.scipy.org/.

16. Y. Arntz, J. D. Seelig, H. P. Lang, J. Zhang, P. Hunziker, J. P. Ramseyer, E. Meyer, M. Hegner, and C. Gerber, "Label-free protein assay based on a nanomechanical cantilever array", Nanotechnology 14, 86 (2003).

17. S. Dante, D. Duval, D. Fariña, A. B. González-Guerrero, and L. M. Lechuga, "Linear readout of integrated interferometric biosensors using a periodic wavelength modulation", Laser Photonics Rev. (2015).

\section{Introduction}

The development of transducers to be part of a biosensor device is specially focused in the achievement of high sensitive ones for real-time monitoring. These two conditions are highly fulfilled in the case of nanomechanical transducers, becoming a valuable sensing platform for many different applications, ranging from environmental, to chemical or biological [1]. The sensitivity of nanomechanical transducers is controlled by several factors such as its mass, damping (energy dissipation) and stiffness. Depending on the method of operation, static or dynamic, different parameters must be optimized. Beside the intrinsic transducer sensitivity, the final sensitivity of the biosensor will depend on the readout method of the nanomechanical response. Laser beam reflection, piezoelectric or piezoresistive are probably the most common employed readout methods. An alternative method, where a microcantilever forms a waveguide in which light propagates, was presented for the first time by our research group few years ago [1]. The proposed opto-mechanical device is a combination of the well-known nanomechanical transducer method and the integrated optical technology, and it was called optical waveguide microcantilever (OWC). The light propagated through the microcantilever is emitted from the free end, travels across a small gap, and is captured by an output single mode waveguide. Following this initial work, different approaches combining photonic and microcantilever devices have been developed, looking for a higher integration of arrays of microcantilevers with a high sensitive readout method. Highly integrated opto-mechanical sensors based on silicon [2,3] or polymer technology [4,5] have been presented. However, the sensing applications demonstrated with this method are still few [6,7], and the sensitivities achieved are lower than the ones reached with the laser beam reflection method.

In this paper, we present an exhaustive optical and mechanical analysis of photonic microcantilevers in order to improve their sensitivity to be used as a biosensor device. Working on the surface stress mode, the rise on the sensitivity is achieved by increasing the microcantilever deflection during a sensing process, while reducing the signal to noise ratio. On the other hand, the final sensitivity of the OWC depends on the minimum intensity change that can be detected due to the microcantilever deflection. This study shows the analysis of the parameters that have a direct effect on the OWC power efficiency and final sensitivity, and determine the optimal values to maximize both the microcantilever deflection and power change. The microcantilever thickness is one of the key parameters studied due to its impact on the mechanical and optical behavior. Other parameters such as the output waveguide thickness and the gap distance, and the dimensions at the interface between the input waveguide and the OWC were also analyzed.

\section{Photonic microcantilever transducer method}

The OWC working principle is based on the optical coupling efficiency change between two total internal reflection (TIR) waveguides. Fig. 1.a shows a scheme of the device configuration. The first waveguide is a silicon dioxide microcantilever (OWC), and the second one is an output silicon nitride waveguide $(\mathrm{OW})$. Both waveguides are separated by a gap distance, $L_{g}$. The light transmitted from the OWC to the OW changes dramatically with the transversal displacement of the $\mathrm{OWC}$ free end. This displacement is induced by biomolecular interactions occurred on one side of the OWC (Fig. 1.c), given by the surface stress difference between the OWC surfaces. This displacement can be determined by reading the output power changes in the OW using a photodetector. The light reaches the OWC from 
an input silicon nitride waveguide (IW), where the light is introduced from a laser source using an optical objective.

The microcantilever is vertically displaced respect to the IW and OW (see Fig. 1.b). This configuration has two main effects: a) the light propagating through the IW is mainly coupled into de OWC by the evanescent field which travels through the silicon oxide cladding; $b$ ) it produces a non-symmetric optical response curve of the OWC respect to its initial position (cero deflection). With this configuration, and due to the gaussian profile of the optical response curve, the movement of the microcantilever during the biorecognition process will take place in a high sensitivity region of the optical response curve, allowing as well to know the direction (up or down) of the OWC deflection.

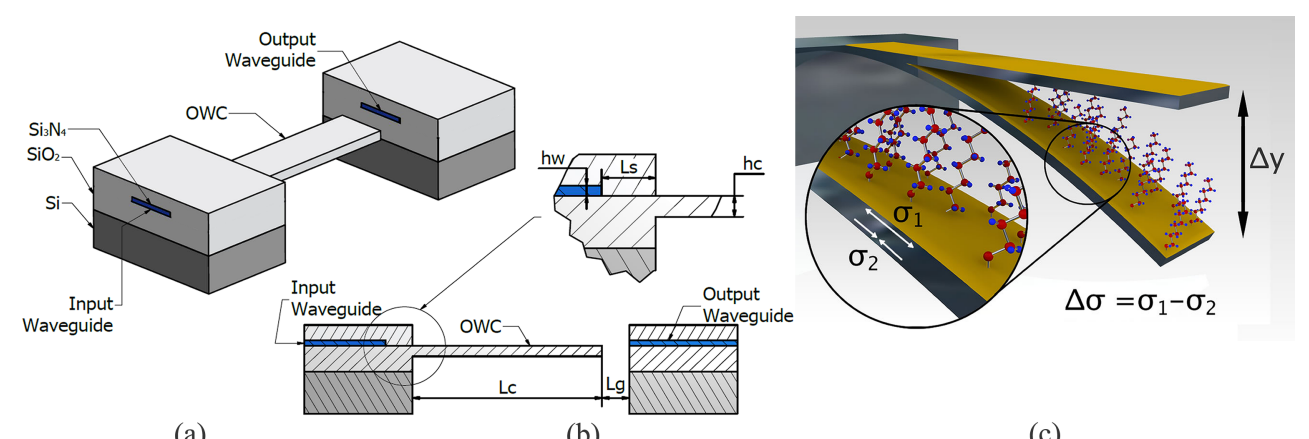

(a)

(b)

(c)

Fig. 1. Scheme of the optical waveguide cantilever device: a) OWC 3D view, b) OWC vertical cross-section c) Microcantilever bending by surface stress.

\subsection{OWC design}

The OWC device is a silicon dioxide microcantilever, with length, $L_{c}$, thickness, $h_{c}$, and width, $b_{c}$, which acts as the core of a rectangular waveguide where the light is confined, where the external medium acts as the claddings (see Fig. 1b).

The IW and OW have a rectangular silicon nitride core, surrounded by silicon dioxide claddings, which make a symmetric waveguide. Both cores have the same dimensions: thickness, $h_{w}$, and width, $b_{w}$. The IW and OW have an important role in the total device output power efficiency and the device sensitivity.

The end of the IW and the clamped side of OWC are separated horizontally by a protection distance, $L_{s}$. This protection distance avoids additional extra stress in the clamp of the microcantilever, due to the silicon oxide - silicon nitride interface and the gradient stress that could be generated by the deposition of the silicon nitride layer. The $L_{s}$ distance must be optimized to achieve the maximum coupling of light between the IW and the OWC, and, therefore, to maximize the output power efficiency.

The OW is separated few microns from the free end of the OWC by a gap distance $L_{g}$. The gap distance has a direct effect in the optical sensitivity and the maximum deflection amplitude that the OW can read.

In order to increase the sensitivity and the output power efficiency we have studied how each of the above dimensions affects the OWC behavior. The main aim is to increase the mechanical deflection and to maximize the output power change in relation with the mechanical bending.

\subsection{Theory and modelling}

During a biosensing assay, biomolecular interactions occur on one side of OWC and a differential surface stress, $\Delta \sigma$, between the two OWC surfaces is produced, inducing the displacement, $\Delta y$, of the OWC free end. The deflection distance $\Delta y$ can be approximated by the Stoney equation, including the effect of the clamping by [8]: 


$$
\Delta y \approx \frac{4(1-v)}{E}\left(\frac{L_{c}}{h_{c}}\right)^{2} \Delta \sigma
$$

Where $v$ is the Poisson ratio, $E$ is the Young modulus, and $L_{c}$ and $h_{c}$ are the length and the thickness of the microcantilever, respectively.

Working on the static mode, the minimum deflection that could be detected would also depend on the microcantilever thermal noise (Brownian motion). The microcantilever dimensions must be chosen in order to achieve a high signal to noise ratio. The microcantilever vibration is characterized by its resonance frequency for a specific vibration mode, $w_{n}$, and its quality factor, $Q_{n}$. The thermal noise amplitude, $\Delta y_{\min }$, can be obtained from Eq.(2), derived from the equipartition theorem, where the average energy of a system in thermal equilibrium is the same for each vibration mode:

$$
\Delta y_{\min }=\sqrt{\frac{2 k_{b} T \Delta w}{\pi Q_{n} w_{n}}}
$$

Where $k_{b}$ is the Boltzman constant, $T$ is the temperature, and $\Delta w$ is the bandwidth. The resonance frequency derived from the Euler-Bernoulli beam theory [9] is given by [10]:

$$
w_{n} \cong \alpha_{n}^{2} \frac{h_{c}}{L_{c}^{2}} \sqrt{\frac{E}{12 \rho_{c}}}
$$

Where $\rho_{c}$ is the microcantilever density and $\alpha_{n}$ takes values of $1.875,4.694,7.588, \ldots$ for the first three vibration modes [11].

Finally the Q factor for each vibration mode can be obtained from Hosaka et. al. approximation, where the microcantilever is modeled as a line of spheres with diameter $b$, equals to the microcantilever width, surrounded by external medium with density, $\rho_{m}$, and viscosity, $v[12]$ :

$$
Q_{n}=\frac{\rho_{c} h_{c} b^{2} w_{n}}{3 \pi \eta b+\frac{3}{4} b^{2} \sqrt{2 \rho_{m} \nu w_{n}}}
$$

The proposed opto-mechanical device is at the same time based on dielectric waveguides, where the light is confined by Total Internal Reflection (TIR) into a medium with high refractive index (the core), surrounded by mediums of lower index (the claddings). According to Maxwell theory, light can propagate in such structure in the form of guided modes, characterized by their effective refractive index. For a Transversal Electric polarization (TE), the solution of the wave equation depends on the wavelength, the refractive index of the core and claddings, and by the propagation constant. By applying the boundary conditions ( $\mathrm{y} \rightarrow \pm \infty$ ), and by imposing the continuity of $E_{y}$ and $d E_{y} / d x$ at the interfaces, we can obtain the eigenvalue equation for the TE modes for asymmetric planar waveguides:

$$
\mathrm{V} \sqrt{1-b}=m \pi+\tan ^{-1}\left(\sqrt{\frac{b}{1-b}}\right)+\tan ^{-1}\left(\sqrt{\frac{b-a}{1-b}}\right)
$$

Where, $b$ is the normalized effective refractive index, $\mathrm{V}$ is the normalized thickness of the waveguide, and $a$ is the asymmetry of the slab waveguide.

From Eq.(5) it is possible to calculate the $E_{z}$ component of the electric field in each region of the slab waveguide: 


$$
E_{z}(y)= \begin{cases}A\left(\cos \left(k_{y} d\right)+\frac{\gamma_{c}}{k_{y}} \sin \left(k_{y} d\right)\right) e^{-\gamma_{c}(y-d)} & y>d \\ A\left(\cos \left(k_{y} y\right)+\frac{\gamma_{c}}{k_{y}} \sin \left(k_{y} y\right)\right) & 0<y<d \\ A e^{-\gamma_{s} y} & y \leq 0\end{cases}
$$

Where $k_{y,}, \gamma_{c}$ and $\gamma_{s}$ are the transverse propagation coefficients for the core, cladding and substrate, respectively and $d$ is the core thickness. The electric field shows a sinusoidal dependence in the core and exponentially decreases in the claddings (evanescent field). The constant A represents the field amplitude, and is related to the energy carried by the mode.

When a specific mode changes from one waveguide to another, the coupling coefficient for each mode into the second waveguide can be obtained by the overlapping integral [13]:

$$
\eta=\frac{\left(\int_{-\infty}^{\infty} A_{n}(y) B_{m}^{*}(y) d y\right)^{2}}{\int_{-\infty}^{\infty} A_{n}(y) A_{n}^{*}(y) d y \int_{-\infty}^{\infty} B_{m}(y) B_{m}^{*}(y) d y}
$$

Where $A_{n}$ is the amplitude distribution of the mode $n$ in the first waveguide, and $B_{m}$ is the amplitude distribution of the mode $m$ in the second waveguide.

\subsection{OWC sensitivity}

The final sensitivity of the OWC device is the combination of the mechanical and optical sensitivity. The interrelation of the different OWC parameters that affects each sensitivity and the output power efficiency is shown in Fig. 2.

The mechanical sensitivity is related with the OWC free end deflection when the biomolecular interactions occur on its surface. This sensitivity depends on the material and dimensions of the OWC, as can be observed on the Stoney equation. According with this model, beside the material properties, the main physical parameters that govern the mechanical response are the OWC length, $L_{c}$, and thickness, $h_{c}$. Higher microcantilever deflections would be achieved for longer and thinner microcantilevers.

On the other hand, the optical sensitivity, $S$, is defined as the optical coupling efficiency, $\eta$, change between the OWC and the OW per unit of the microcantilever displacement:

$$
S=\frac{\partial \eta}{\partial z}
$$

Thus, the optical sensitivity depends on the OWC and OW dimensions. As shown in Fig. 2 , the OWC thickness is the critical dimension of the device because it affects both the mechanical and optical sensitivities. Finally, regarded with the total output efficiency the distance between the IW and the OWC must also be considered.

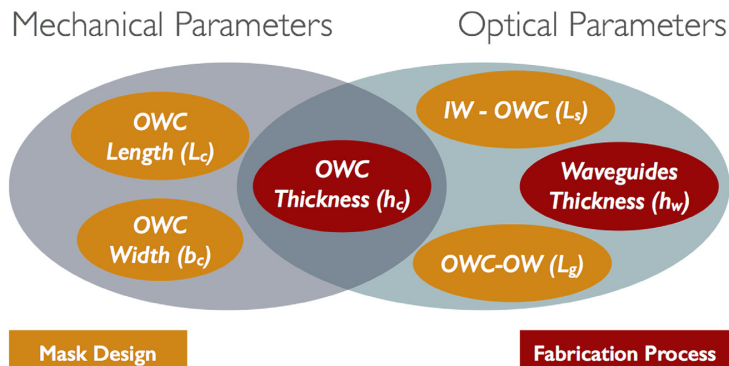

Fig. 2. Classification of the OWC dimension parameters related with the optical and mechanical sensitivity and the output power efficiency. 


\section{Numerical analysis}

Numerical simulations in COMSOL multiphysics [14] were done to obtain the optimal device dimensions. We studied how each dimension affects to the OWC behavior to maximize the mechanical deflection and also to know the maximum optical output power changes in relation with the mechanical bending.

To verify the initial results for the optical and mechanical equations, analytical calculations were done using python scripts with scipy library [15], which helped us to compare the analytical results with the COMSOL simulations.

The selected material for the microcantilever is silicon dioxide $\left(\mathrm{SiO}_{2}\right)$ with a refractive index of $n_{\mathrm{SiO} 2}=1.46$, Young modulus $E=70 \times 10^{9} \mathrm{~Pa}$ and Poisson ratio of $v=0.17$. The microcantilever acts like a symmetric waveguide, where the surrounding medium, air $\left(n_{\text {air }}=1\right)$ or water $\left(n_{\text {water }}=1.33\right)$, acts like a cladding.

For the IW and the OW core the selected material is silicon nitride, with a refractive index of $n_{S i 3 N 4}=2.00$. The selected material for the surrounding cladding layers is silicon dioxide $\left(\mathrm{SiO}_{2}\right)$ with a refractive index of $n_{\mathrm{SiO} 2}=1.46$.

The simulations were executed in a computer with 4 cores processor (Intel Xeon E5520) working at $2.27 \mathrm{GHz}$ clock frequency and 24 GB RAM memory, running at Windows 2008 server operating system.

\section{Results and discussion}

\subsection{OWC Mechanical behavior}

The simulation of the relationship between the microcantilever free end bending and the microcantilever thickness for lengths between $100 \mu \mathrm{m}$ and $500 \mu \mathrm{m}$ was made for a maximum difference surface stress of $\Delta \sigma=0.05 \mathrm{~N} / \mathrm{m}$ [16].

The employed physical model is shown in Fig. 3.a. A silicon dioxide rectangular beam, with dimensions $L_{c}, h_{c}$ and $b$, is clamped to a fixed block of the same material. A homogeneous rectangular mesh was selected for the microcantilever domain and a tetrahedral mesh for the fixed block, which reduced the processing time.

For the bending study a stationary analysis was performed by applying a longitudinal force $F$ (see Fig. 3.a) in the microcantilever free end. The value for the microcantilever bending can be obtained from any free end point, shown in Fig. 3.b. The microcantilever bending increases for thinner and longer structures. The comparison between the analytic solutions of Eq. (1), and the COMSOL simulations gave differences lower than $1 \%$.

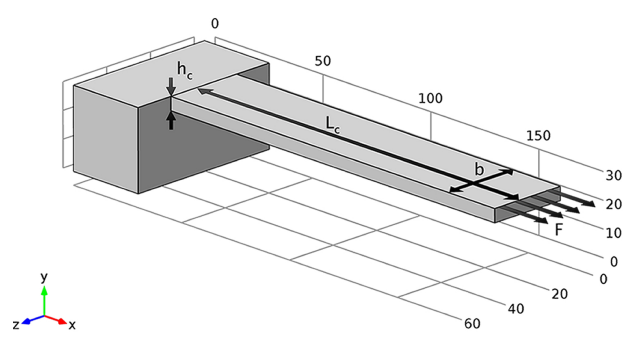

(a)

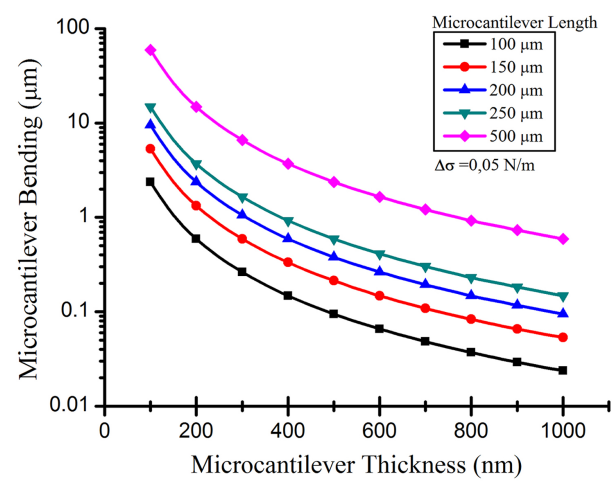

(b)

Fig. 3. Physical model for microcantilever mechanical studies: a) mechanical model used for COMSOL simulations b) microcantilever free end displacement as function of its thickness, for a range of $100 \mu \mathrm{m}$ to $500 \mu \mathrm{m}$ of length, for a differential surface stress of $\Delta \sigma=0.05 \mathrm{~N} / \mathrm{m}$. 
The microcantilever resonance frequency was obtained from an Eigen-frequency study applied to the model. For the fundamental vibration mode, the difference between COMSOL simulations and analytical values derived from Eq.(3) were lower than $1 \%$. These results provide a good level of confidence of the mechanical model.

\subsection{Optical behavior: waveguides propagation modes}

The proposed design has three waveguides: two identical silicon nitride core waveguides (IW and $\mathrm{OW}$ ), and a silicon dioxide microcantilever (OWC). The number of guided modes that will propagate along these waveguides is determined by the relation of the effective refractive index, $N_{\text {eff }}$, of each waveguide with its thickness.

We considered the waveguides as symmetric slabs, because the high relation between its width and thickness. The physical model consists in a slab waveguide section, with an input in $\mathrm{x}=0$, where the electric field profile $E_{z}(y)$ is calculated for a TE polarization. For the optical simulations, the mesh size must be at least $\lambda / 3$. We chose to use a mesh with a rectangular distribution in the core, and a triangular distribution for the cladding layers, for $\lambda=660 \mathrm{~nm}$. Solving the dispersion Eq.(5) for the TE modes, the effective refractive index for each propagation mode, $m$, as a function of the waveguide thickness was obtained. The effective refractive index for the input and output waveguides is shown in Fig. 4.a. For the OWC we analyzed two different conditions: when the microcantilever is surrounded by air $\left(n_{\text {air }}=1\right)$ and by water $\left(n_{\text {water }}=1.33\right)$, shown in Fig. $4 . \mathrm{b}$ and c, respectively. In both cases, the number of propagation modes increased with the thickness of the waveguide.

The theoretical cut-off for the single mode behavior for TE modes can be derived making $N_{\text {eff }}=n_{s}$, so $b \rightarrow 0$. Operating with the dispersion Eq.(5), the value of the cut-off thickness for the number of $m$ modes that can propagate can be written as:

$$
\mathrm{V}_{T E}=m \pi+\tan ^{-1} \sqrt{a}
$$

As the three waveguides are symmetric, the fundamental mode always exist, thus the maximum thickness for a single mode behavior is $h_{w}=241.42 \mathrm{~nm}$ for IW and OW, and $h_{c}=$ $310.22 \mathrm{~nm}$ and $h_{c}=554.09 \mathrm{~nm}$ for the OWC in air and water, respectively.
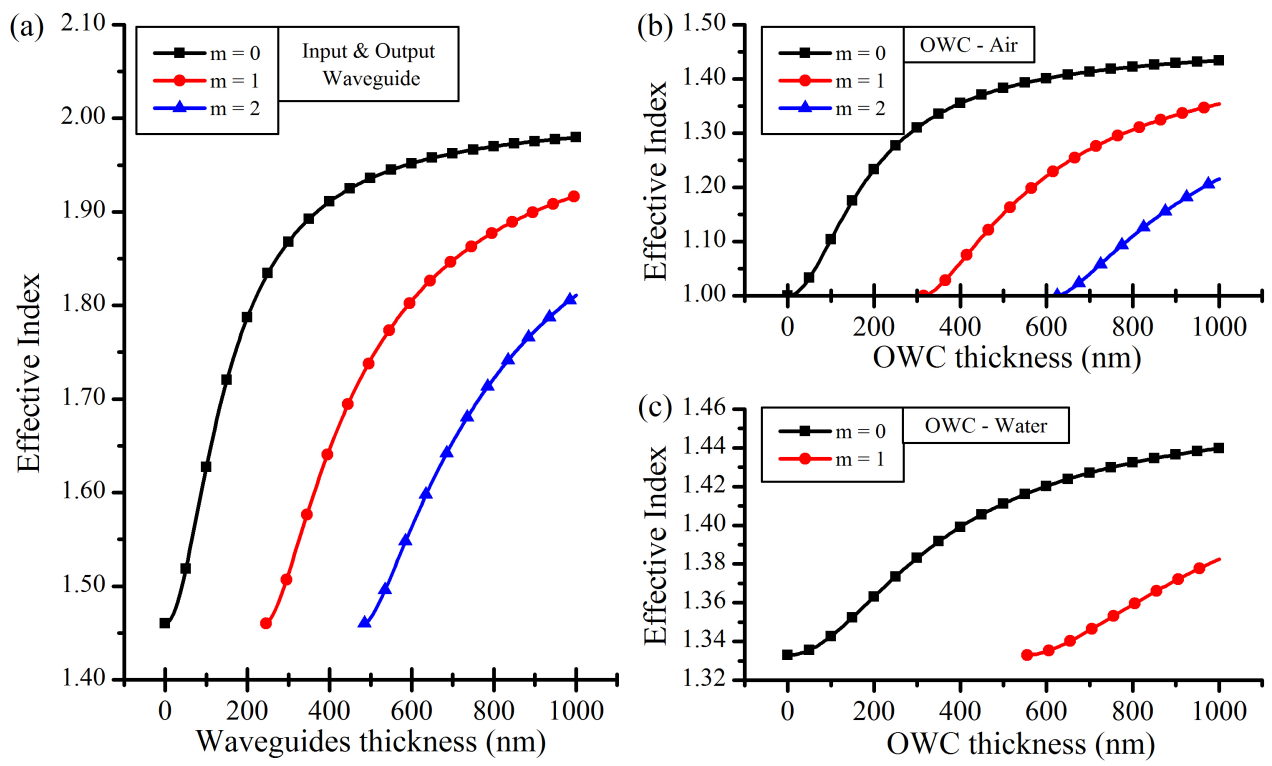

Fig. 4. Dependency of the effective refractive index of each waveguide as a function of the thickness: a) IW and OW, b) OWC in air and c) OWC in water. 


\subsection{Optical models}

The optical propagation in the device was analyzed in two steps. Initially, a physical model including only the IW-OWC (see Fig. 5.a), interface was considered, with the aim to find the values that maximize their coupling efficiency. Next, the OW was included, to simulate the whole device behavior (see Fig. 5.b).

In both cases, the physical model is surrounded by a perfect Matching Layer (PML) to absorb the electromagnetic field in the model boundaries and to avoid reflections. The meshing strategy is based on rectangular elements in the IW, OW and OWC with a maximum element size of $41.25 \mathrm{~nm}(\lambda / 16)$.

The efficiency, $\eta$, of the device is defined as the percentage of the introduced optical power that reaches the output:

$$
\eta=10^{S_{21}(d B)} / 10
$$

The parameter $S_{21}$ is defined as the optical power ratio at the output. The input light source is a TE fundamental mode, with $1.0 \mathrm{~W}$ power. Assuming this power, the interface IWOWC efficiency can be obtained from Eq.(10).

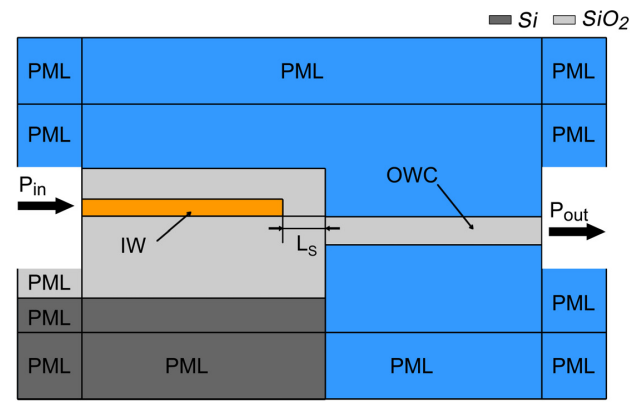

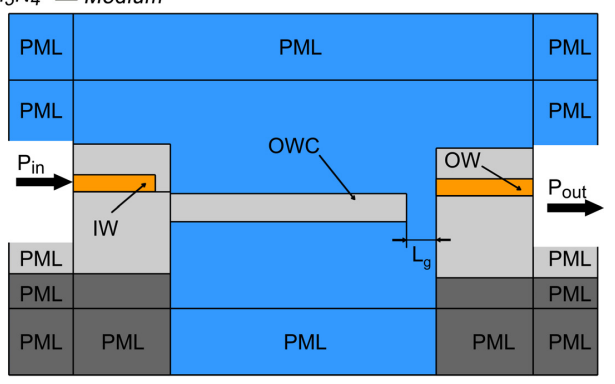

b)

Fig. 5. Physical models used for COMSOL simulations: a) model used in the study of the IW and OWC interface to increase the optical coupling efficiency into the OWC b) model used in the study of devices sensitivity and device power efficiency.

\subsection{IW and $O W C$ interface}

The light from the IW is coupled into the OWC at the microcantilever clamping region. To maintain a fabrication process as simple as possible, the IW has a rectangular shape (Fig. 5.a). This interface between the IW and the OWC has a critical influence in the device output optical power and it has an impact in the signal to noise ratio.

To improve the overall device power efficiency, we studied the output power at the free end of the OWC for three different IW thicknesses $(80 \mathrm{~nm}, 130 \mathrm{~nm}$ and $180 \mathrm{~nm})$ and an OWC thickness range from $100 \mathrm{~nm}$ to $1 \mu \mathrm{m}$.

The results of this study for air and water as external medium are shown in Fig. 6.a and b, respectively, where the efficiency of each propagation mode is calculated for each OWC thickness. The coupling efficiency increase for smaller IW thicknesses, due to a deeper penetration of the evanescent field of the fundamental mode as the IW thickness is reduced. Attending to the OWC thickness, the maximum efficiency occurs when the OWC has single mode behavior. For OWC thickness higher than the cut-off thickness, the power is split between the propagation modes, which decrease the coupling efficiency of the fundamental mode, generating a more complex response, as shown in section 4.7. Therefore, the maximum coupling efficiency of the fundamental mode occurs for OWC thickness around $200 \mathrm{~nm}$ in water (Fig. 6.b). Instead, this cut-off thickness is under $100 \mathrm{~nm}$ when surrounded by air (Fig. 6.a). The electric field representation, for water as external medium, is shown in the insets of Fig. 6.b, where it is observed the higher coupling of light when only the fundamental mode is 
guided (inset on the left). In the case of two guided modes, it is possible to observe the interference between both propagation modes along the microcantilever (inset on the right), producing a spatial displacement of the output response at the microcantilever free end.

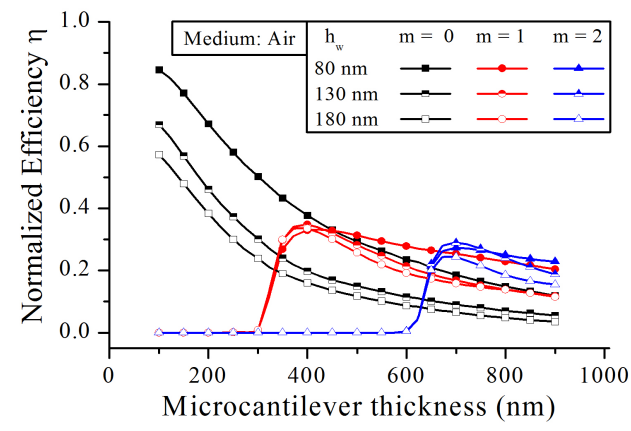

(a)

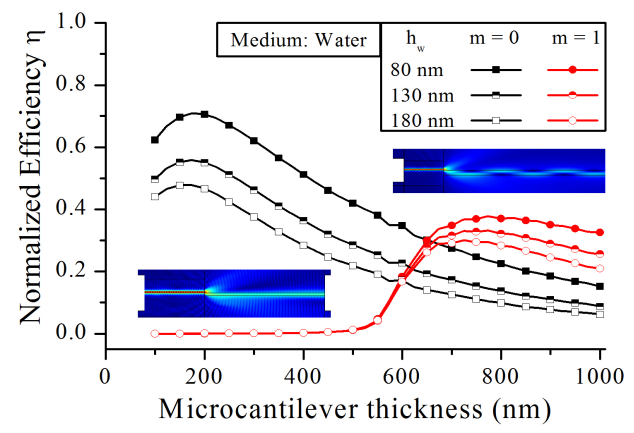

(b)

Fig. 6. OWC-IW interface efficiency as a function of microcantilever thickness for a specific external medium (a) efficiency for air as external medium and, (b) efficiency for water as external medium.

\subsection{IW-OWC interface (safety distance)}

Another parameter that could affects the light coupling between the IW and the OWC is the distance between the IW end and the microcantilever clamp. This distance of few nanometers is named security distance, $L_{s}$ (Fig. 5.a). From a fabrication point of view, the security distance has a positive effect, because it reduces the possible stress induced over the OWC during the deposition process of the silicon nitride, leaving the OWC free of initial stress. For studying the effect of the safety distance, we used the previous physical model (Fig. 5.a).

The result when water is a external medium is shown in Fig. 7, where the relation between the coupling efficiency and the $L_{s}$ is obtained for an IW $130 \mathrm{~nm}$ thick. We can observe that for microcantilevers thicker than $200 \mathrm{~nm}$, the coupling efficiency of the fundamental mode is slightly improved for $L_{s}=0.5 \mu \mathrm{m}$ (respect $L_{s}=0 \mu \mathrm{m}$ ).

For larger $L_{s}$, the efficiency has a lower dependence with the OWC thickness, due to a change in the power transmission mechanics during the light coupling. For smaller $L_{s}$ the light is mainly coupled through the evanescent field, while for large distances the direct coupling is the principal mechanism. The same effect occurred for air as external medium. Looking to achieve an increase of the power efficiency for OWC with single mode behavior, the optimal $L_{s}$ distance selected was $0.5 \mu \mathrm{m}$.

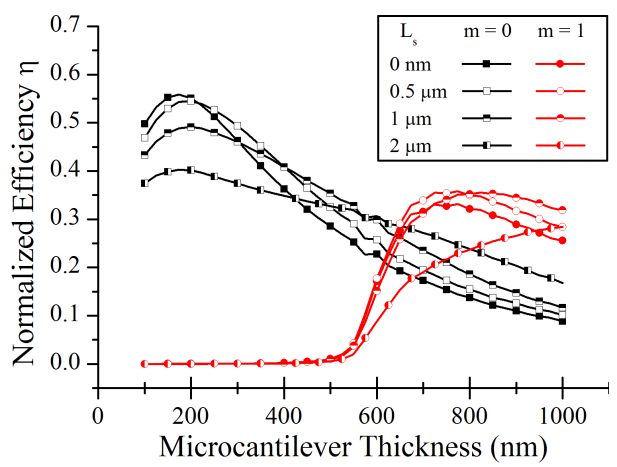

Fig. 7. Normalized efficiency as fuction of OWC thickness for different distances between the IW end and the microcantilever clamp. 


\subsection{Gap effect-Static Response}

The OWC free end is separated from the output waveguide (OW) by a few nanometers, called the gap distance $\left(L_{g}\right)$. This distance has a strong influence in the OWC sensitivity and in the maximum detectable bending range. To exploit the final sensitivity of the device, we performed a static analysis, including the influence of the OWC thickness in the device efficiency, for two difference gap distances and two waveguide thicknesses. The employed physical model included all the elements: IW, OWC and OW (Fig. 5.b), with an initial position of cero deflection for the OWC. For simplicity in the fabrication process, the OW and IW have the same thickness, are single mode, and both are protected by $1 \mu \mathrm{m}$ silicon dioxide layer to avoid propagation losses due to light scattering.

The relation between the total efficiency at the end of the OW as a function of the OWC thickness for gap distances $L_{g}=1 \mu \mathrm{m}$ and $L_{g}=2 \mu \mathrm{m}$ is plotted in Fig. 8. The total efficiency is obtained from Eq. (10), using the previously calculated scatter parameter $S_{21}$ for the fundamental mode at the end of the OW. Water is considered as external medium, with an IW and OW thickness of $130 \mathrm{~nm}$ and $180 \mathrm{~nm}$, respectively. The modal behavior as function of the OWC thickness is clear for both $L_{g}$ distances. For OWC thickness lower than $500 \mathrm{~nm}$ the single mode behavior has a maximum efficiency zone in the range of $175 \mathrm{~nm}$ up to $300 \mathrm{~nm}$, with a maximal total device efficiency around $25 \%$ for a $130 \mathrm{~nm}$ waveguide with a $L_{g}=1 \mu \mathrm{m}$. For waveguides thicknesses of $180 \mathrm{~nm}$, the total device efficiency is around $20 \%$, both for $L_{g}$ $=1 \mu \mathrm{m}$ and $L_{g}=2 \mu \mathrm{m}$, given that the increased $L_{g}$ distance is compensated by the higher OW thickness.

For OWC thickness higher than $500 \mathrm{~nm}$ the multimode behavior creates constructive and destructive zones that affect the output power. This effect is undesirable because any change in the refractive index of the external medium will be translated into a variation of the effective refractive index of the propagating mode, which could hide or modify any change on the optical power due to a biorecognition process. This effect is discussed more in detail in section 4.8.

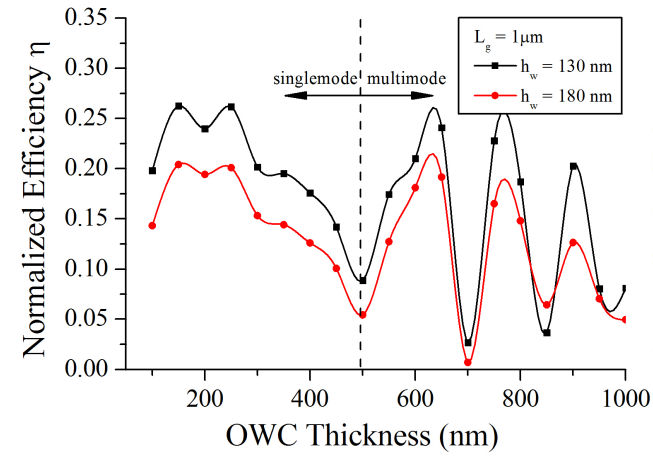

(a)

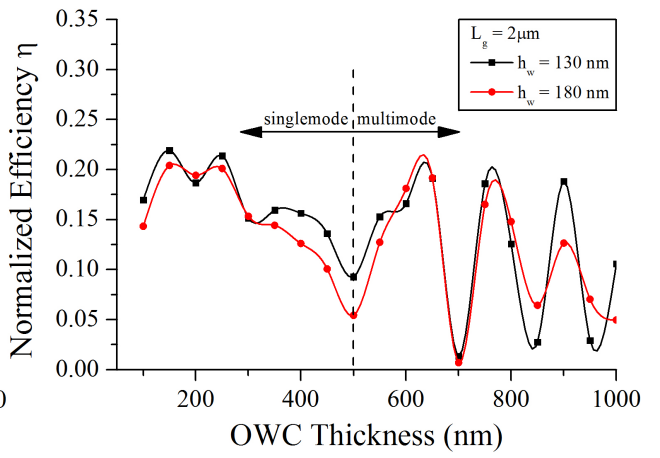

(b)

Fig. 8. Total output efficiency: a) gap distance of $1 \mu \mathrm{m}$ b) gap distance of $2 \mu \mathrm{m}$.

\subsection{Effect of cantilever thickness on the free-end displacement}

The OWC bending response, and the change of light power coupled into the OW during its deflection, determines the device sensitivity. Trying to obtain a realistic behavior of the device, we used the same physical model (Fig. 5.b), combining the physics of solid mechanics and the electromagnetic waves in COMSOL. 


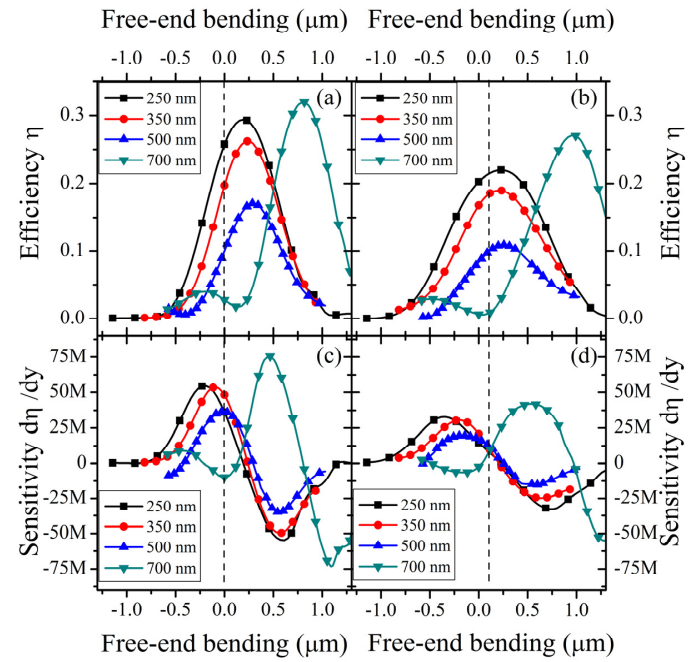

Fig. 9. OWC bending response for: a) $\mathrm{L}_{\mathrm{g}}=1 \mu \mathrm{m}$ and b) $\mathrm{L}_{\mathrm{g}}=2 \mu \mathrm{m}$. OWC sensitivity for: c) $\mathrm{L}_{\mathrm{g}}=1 \mu \mathrm{m}$ and d) $\mathrm{L}_{\mathrm{g}}=2 \mu \mathrm{m}$.

We studied the change in the coupling efficiency when a force is applied at the end of the OWC, mimicking a change of the surface stress of the microcantilever. After the OWC is bended, a COMSOL frequency study is applied to calculate the light propagation in the device during the OWC deflection. Varying the applied force, the relationship between the displacement of the free end and the total efficiency was obtained. Fig. 9.a and b show the coupling efficiency for the corresponding OWC bending for different microcantilever thicknesses and gap distances of $1 \mu \mathrm{m}$ and $2 \mu \mathrm{m}$, respectively. Directly applying the derivative of these curves, the device sensitivity was obtained in Fig. 9.c and d.

The benefits of the misalignment between OWC and OW for zero OWC displacement can be observed in Fig. 9.c and d, respectively, where any change of power due to the microcantilever bending occurs in the region of the curve of maximum slope. This means that the misalignment configuration will display higher changes of the power coupled into the OW for the same microcantilever bending, and, therefore, will display better sensitivities than if they were perfectly aligned, in spite of the decrease in the total coupled efficiency. Fig. 9.a and $b$ shows as well the effect of the gap distances. Gap distances of $1 \mu \mathrm{m}$ show narrower Gaussian profiles of the coupled efficiency than gaps of $2 \mu \mathrm{m}$. In contrast, the microcantilever deflection range that could be measured decrease with the shorter gaps. For that reason, the selection of the gap distance is a compromise between the sensitivity and the maximal measurable OWC deflection.

Table 1. Optical Initial efficiency and OWC deflection for 3db coupling losses, IW and OW thickness $130 \mathrm{~nm}$, water as external medium

\begin{tabular}{lccccc}
\hline & \multicolumn{2}{c}{ Initial efficiency } & & \multicolumn{2}{c}{$3 \mathrm{~dB}$ Displacement } \\
\cline { 2 - 3 } \cline { 5 - 6 } OWC thickness $\left(h_{c}\right)$ & $L_{g}=1 \mu m$ & $L_{g}=2 \mu m$ & & $L_{g}=1 \mu m$ & $L_{g}=2 \mu m$ \\
\hline $250 \mathrm{~nm}$ & 0.258 & 0.203 & & $252 \mathrm{~nm}$ & $381 \mathrm{~nm}$ \\
$350 \mathrm{~nm}$ & 0.197 & 0.168 & & $188 \mathrm{~nm}$ & $297 \mathrm{~nm}$ \\
$500 \mathrm{~nm}$ & 0.102 & 0.084 & & $114 \mathrm{~nm}$ & $221 \mathrm{~nm}$ \\
\hline
\end{tabular}

Table 1 shows the microcantilever deflection that would be necessary to produce $3 \mathrm{~dB}$ change at the output efficiency for each gap distance. The deflections were calculated for devices with IW and OW thicknesses of $130 \mathrm{~nm}$ and water as external medium. For OWC of 
$350 \mathrm{~nm}$ of thickness, the $2 \mu \mathrm{m}$ gap distances required nearly double deflection to produce the same change than for gaps of $1 \mu \mathrm{m}$.

\subsection{Refractive index effect}

The biorecognition process involves changes in the OWC environment, due to changes in the solution where the biomolecules are transported. As the external medium acts as OWC cladding, this influences to the effective refractive index of the guided modes. For OWC with multimode behavior, changes in the external refractive index generate interferences between each propagation mode and, in consequence, in the output power.

To study this influence, we simulated the effect of a refractive index change of the external medium of $6 \times 10^{-2}$ RIU, for three different OWC thicknesses: $250 \mathrm{~nm}, 550 \mathrm{~nm}$ and $700 \mathrm{~nm}$. Changes in the output efficiency are related with the phase change of a sinusoidal signal, and depend on the working wavelength, $\lambda$, the difference between the effective index of the two guided modes, $\Delta N_{\text {eff }}$, and the OWC length, $L_{c}[17]$ :

$$
\Delta \theta=2 \pi \frac{L_{c}}{\lambda} \Delta N_{e f f}
$$

Two different behaviors of the efficiency can be observed with the external refractive index change (Fig. 10.a): for multimode OWC $\left(h_{c}>500 \mathrm{~nm}\right)$ the modes interference produces an oscillation in the efficiency of about $30 \%$. Instead, for single-mode OWC the variation is less than 5\%. For this reason OWC with single mode behavior are preferred, avoiding displacement readouts that could hide or affect the signal produced by the biorecognition process. Similarly, the OWC with bimodal behavior can shift the maximum peak position depending on the external medium, due to the effect of the external medium on the effective refractive index of the guided modes, as shown in Fig. 10.b.

Fortunately, the changes in the refractive index of the medium due to the presence of molecules are much lower than the case shown here. The injection of a specific solution with different concentrations of molecules should not have any effect on the device modal behavior.

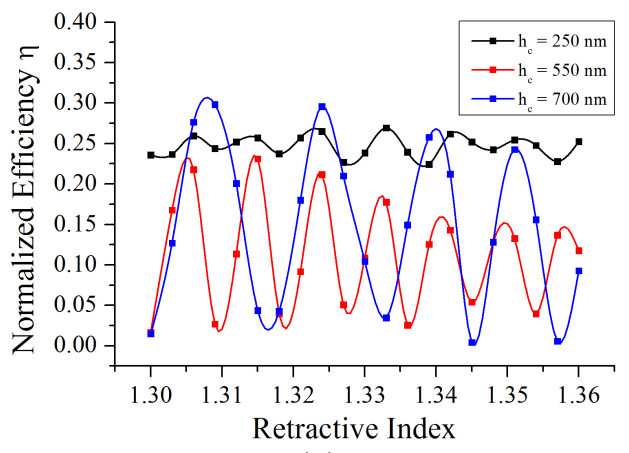

(a)

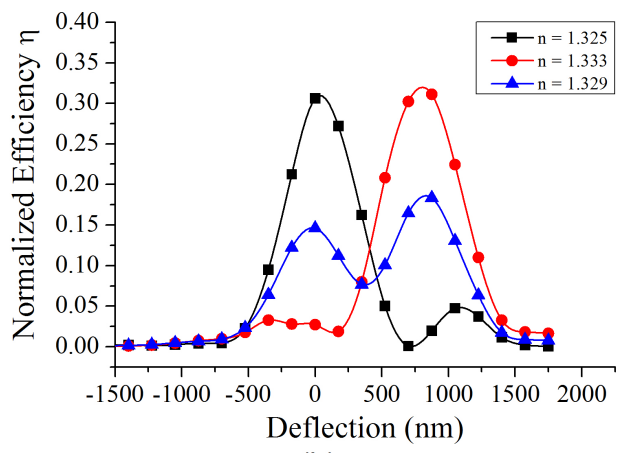

(b)

Fig. 10. Effect of changes in the refractive index of the external medium : a) variation of the total optical power as a function of the refractive index for three microcantilever thickness, b) effect of bimodal OWC response displacement for three external medium with different refractive index.

\subsection{Figure of Merit}

Finally, to characterize the performance of the device relative to its efficiency and sensitivity, we defined the Figure of Merit (FOM) as:

$$
F O M=\eta \frac{\partial \eta}{\partial z}
$$


The FOM value for two waveguide thicknesses, $h_{w}=130 \mathrm{~nm}$ and $h_{w}=180 \mathrm{~nm}$, and for gap distances $L_{g}=1 \mu \mathrm{m}$ and $L_{g}=2 \mu \mathrm{m}$, is shown in Fig. 11. In all cases a microcantilever thickness range between $150 \mathrm{~nm}$ up to $400 \mathrm{~nm}$ meets with the best efficiency response with a high sensitivity. However, a large difference is observed between both $L_{g}$ distances given by the higher efficiency and sensitivity for devices with $L_{g}=1 \mu \mathrm{m}$.

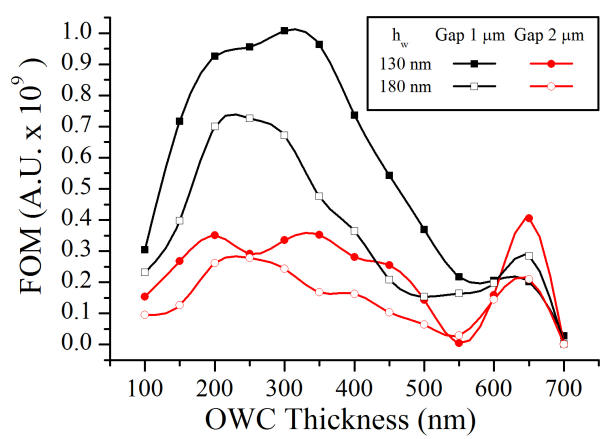

Fig. 11. Figure of merit to select the optimum OWC thickness.

\section{Conclusions}

We have analyzed the main parameters that affect the final performance of the OWC devices, in order to improve their surface stress sensitivity and maximize their optical response to be employed as a reliable biosensor. We have studied both the mechanical and the optical response for different waveguides and microcantilever thicknesses, external medium and gap distances. We obtained that a OWC thickness in the range of 150-400 nm produces an increment of the sensitivity, motivated by a high mechanical response to the surface stress, as well as high optical response because its single mode behavior. Besides to OWC thickness, the gap distance of $1 \mu \mathrm{m}$ and $2 \mu \mathrm{m}$ between the $\mathrm{OWC}$ and the output waveguide is analyzed, in order to obtain the relation between the output power changes with the free end OWC movement and the maximum detectable deflection distance. To maximize the total output power, the OWC and input waveguide interface was studied, the distance between both has a high impact in the output power, selecting a $500 \mathrm{~nm}$ as an optimum distance to maximize the device output signal and, therefore, its signal to noise ratio.

Biomolecular interactions can generate refractive index changes on the transducer surface, for this reason the device response to the refractive index changes of the external medium has been analyzed for the selected dimensions. Despite a clear dependence of the optical response with the changes of the refractive index, the optical power variation is lower than $5 \%$ for single mode $\mathrm{OWC}$, and $30 \%$ for multimodes $\mathrm{OWC}$, due to modals interferences.

In order to select the optimal dimensions that generate the maximum sensitivity and maximum output power, a figure of merit has been used. These dimensions will be taken into account in the design of the subsequent fabrication process in our clean room facilities, in order to obtain devices that will be used as opto-nanomechanical transducers for surface stress biosensors of high sensitivity.

\section{Acknowledgments}

Authors acknowledge the financial support of Generalitat de Catalunya (2014/SGR/624) and CIBER-BBN. CIBER-BBN is an initiative funded by the VI National R\&D\&i Plan 20082011, Iniciativa Ingenio 2010, Consolider Program, CIBER Actions and financed by the Instituto de Salud Carlos III with assistance from the European Regional Development Fund. 